\title{
Analyzing the propagation of EUV waves and their connection with type II radio bursts by combining numerical simulations and multi-instrument observations ${ }^{\star}$
}

\author{
A. Koukras ${ }^{1,2}$, C. Marqué ${ }^{2}$, C. Downs ${ }^{3}$, and L. Dolla ${ }^{2}$ \\ 1 KU Leuven, Celestijnenlaan 200b - box 2400, 3001 Leuven, Belgium \\ e-mail: alexandros.koukras@kuleuven.be \\ 2 Solar-Terrestrial Centre of Excellence, Royal Observatory of Belgium, Avenue Circulaire 3, 1180 Brussels, Belgium \\ e-mail: christophe.marque@oma.be, laurent.dolla@oma.be \\ 3 Predictive Science Inc., 9990 Mesa Rim Road, Suite 170, San Diego, CA 92121, USA
}

Received 19 June 2020 / Accepted 28 July 2020

\begin{abstract}
Context. EUV (EIT) waves are wavelike disturbances of enhanced extreme ultraviolet (EUV) emission that propagate away from an eruptive active region across the solar disk. Recent years have seen much debate over their nature, with three main interpretations: the fast-mode magneto-hydrodynamic (MHD) wave, the apparent wave (reconfiguration of the magnetic field), and the hybrid wave (combination of the previous two).

Aims. By studying the kinematics of EUV waves and their connection with type II radio bursts, we aim to examine the capability of the fast-mode interpretation to explain the observations, and to constrain the source locations of the type II radio burst emission. Methods. We propagate a fast-mode MHD wave numerically using a ray-tracing method and the WKB (Wentzel-Kramers-Brillouin) approximation. The wave is propagated in a static corona output by a global 3D MHD Coronal Model, which provides density, temperature, and Alfvén speed in the undisturbed coronal medium (before the eruption). We then compare the propagation of the computed wave front with the observed wave in EUV images (PROBA2/SWAP, SDO/AIA). Lastly, we use the frequency drift of the type II radio bursts to track the propagating shock wave, compare it with the simulated wave front at the same instant, and identify the wave vectors that best match the plasma density deduced from the radio emission. We apply this methodology for two EUV waves observed during SOL2017-04-03T14:20:00 and SOL2017-09-12T07:25:00.

Results. The simulated wave front displays a good qualitative match with the observations for both events. Type II radio burst emission sources are tracked on the wave front all along its propagation. The wave vectors at the ray-path points that are characterized as sources of the type II radio burst emission are quasi-perpendicular to the magnetic field.

Conclusions. We show that a simple ray-tracing model of the EUV wave is able to reproduce the observations and to provide insight into the physics of such waves. We provide supporting evidence that they are likely fast-mode MHD waves. We also narrow down the source region of the radio burst emission and show that different parts of the wave front are responsible for the type II radio burst emission at different times of the eruptive event.
\end{abstract}

Key words. magnetohydrodynamics - Sun: radio radiation - Sun: corona - Sun: flares - waves

\section{Introduction}

First discovered using the EIT (Extreme ultraviolet Imaging Telescope) instrument (Delaboudinière et al. 1995) on board SOHO (Solar \& Heliospheric Observatory) and soon closely associated with coronal mass ejections (CMEs) and flares (Moses et al. 1997; Dere et al. 1997; Thompson et al. 1998, 1999), EUV (EIT) waves are large-scale coronal transients of enhanced EUV (extreme ultra-violet) emission propagating away from eruptive active regions (ARs). With a typical speed of several hundred kilometers per second, the question of their possible relation with Moreton waves observed in $\mathrm{H} \alpha$ (Moreton \& Ramsey 1960; Athay \& Moreton 1961) has been raised from the very beginning. Moreton waves display speeds in the range of 500-2000 $\mathrm{km} \mathrm{s}^{-1}$ (Moreton \& Ramsey 1960), which is almost an order of magnitude greater than the Alfvén velocity in the chromosphere (100 $\mathrm{km} \mathrm{s}^{-1}$; Chen \& Fang 2011).

* Movies are available at https://www . aanda.org
This makes it difficult for Moreton waves to be of chromospheric origin since this would require the existence of an unrealistic, strong shock wave (several times (5-20) the local Mach number), to account for propagation distances up to $500000 \mathrm{~km}$ (Moreton \& Ramsey 1960). The apparent difference in velocity and co-location of EUV and Moreton waves reported early on (Eto et al. 2002) was later on smoothed out by more extensive case studies (Warmuth et al. 2001, 2004a). Specifically, the difference in speeds is explained by the difference in the distances at which the wave signature can be observed from the origin point, and by the deceleration of the disturbance during the initial stage (Warmuth et al. 2001, 2004a; Warmuth \& Mann 2011; Shen \& Liu 2012; Shen et al. 2019).

For many years the different characteristics of EUV waves (speed, stationary fronts, connection with flares, driver, etc.) have been debated (see reviews by Gallagher \& Long 2011; Zhukov 2011; Liu \& Ofman 2014), and especially the mechanism to describe them. Patsourakos \& Vourlidas (2012), in a comprehensive review, presented three main possible 
mechanisms. The first is the "true wave" interpretation, where the EUV wave is a fast-mode magneto-hydrodynamics (MHD) wave (e.g., Thompson et al. 1999; Wang 2000; Wu et al. 2001; Ofman \& Thompson 2002; Veronig et al. 2008; West et al. 2011); the present study is based on this mechanism. The second is the "pseudo-wave" mechanism, which explains the EUV wave not as an actual wave but as an apparent motion due to the expansion of the associated CME envelope and the reconfiguration of the magnetic field; different models have been based on this mechanism, such as the successive field line stretching model (Delannée \& Aulanier 1999; Chen et al. 2005), the successive reconnection model (Attrill et al. 2007), and the current shell model (Delannée et al. 2008). The last mechanism, which is the most widely accepted interpretation based on the newest high spatio-temporal observations (SDO/AIA), is the "hybrid" interpretation which is a combination of the previous two and attributes one slow pseudo-wave and one fast EUVwave component to the observations (Chen et al. 2002, 2005; Zhukov \& Auchère 2004; Downs et al. 2011; Shen \& Liu 2012; Shen et al. 2013; Kumar et al. 2013).

Metric type II radio bursts are another well-known signature of large-scale transients in the corona. Discovered in the late 1940s (Wild \& McCready 1950), they are considered as a signature of coronal shock waves (Nelson et al. 1985; Mann et al. 1995; Cliver et al. 1999), triggered either by solar flares or expanding CMEs (see Nindos et al. 2008 for a review). However, their link to EUV waves is uncertain. On one hand, early surveys of type II radio bursts (Klassen et al. 2000) revealed a close association of type II radio bursts with EUV waves, but on the other hand, the association of all EUV waves with type II radio bursts is rather poor (Biesecker et al. 2002; Nitta et al. 2014). This was confirmed by Warmuth et al. (2004a) and Warmuth et al. (2004b) who suggest that the association holds clearly only for the fastest EUV waves, likely to evolve in full-scale coronal shock waves. Mann et al. (1995) interpreted the properties of a sample of metric type II radio bursts as the signature of either weak supercritical parallel or subcritical quasi-perpendicular shock waves, hinting, in either cases, at rather weak shocks with respect to the ambient Alfvén speed. Recent observations have suggested that coronal and even interplanetary type II radio bursts can form in a quasi-perpendicular configuration (Magdalenić et al. 2014; Krupar et al. 2019), as shown by the burst localization at the interaction of the CME flanks with streamers, but this remains an open question.

Part of the uncertainty on the interpretation of type II radio bursts and their relation with other eruptive manifestations comes from the difficulty to extract meaningful parameters from observations. In most cases, type II radio bursts are only observed on dynamic radio spectra as slowly drifting lanes without spatial information. Since type II radio bursts are plasma emission, the frequency drift can be converted into a spatial drift (of the associated shock) only if a density model is assumed. Typically, simple one-dimensional models derived from whitelight measurements during eclipses are used (Newkirk 1961; Saito et al. 1977) and scaled up or down to fit observations. This results in large uncertainties in the derivation of the shock speed.

In this study we aim to perform a detailed investigation of the association between EUV (EIT) waves and type II radio bursts and to see whether or not both can be described in more realistic coronal models than the ones that have been used so far in this context. We follow the formalism developed by Wang (2000) in the modeling of EIT waves, but depart from it by using a recent MHD model for the ambient corona. We model the propagation of EUV waves as a fast-mode MHD wave and identify the ray paths that are compatible with the drift rates deduced from the radio observations. Although, strictly speaking, we do not treat the wave as a shock (unlike e.g., Afanasyev \& Uralov 2011), our results from comparisons with two different eruptive events are encouraging, showing good agreement with EUV and radio observations. Moreover, the simulations show that in both events, type II bursts form in quasi-perpendicular geometry.

The paper is structured as follows: in Sect. 2, we present the modeling in detail, and in Sect. 3 we apply our model to two eruption events that occurred on 3 April 2017 at the west limb and on 12 September 2017 close to disk center. Finally, the results of our analysis are discussed in Sect. 4 and our conclusions in Sect. 5.

\section{Methodology}

This section presents the framework of the present study, including the theoretical model and the formalism that was used to set up the numerical propagation of an EUV wave, the coronal data that were used as input, and the code along with the techniques to make use of the radio data. Lastly, all the sources of data that were used for the analysis are detailed.

\subsection{Magnetohydrodynamics wave model}

The model for the numerical propagation of a fast-mode MHD wave is derived from the work of Uchida (Uchida 1968, 1970; Uchida et al. 1973), and based on the formalism of Wang (2000).

Uchida proposed that the mechanism behind Moreton waves is the propagation of a fast-mode MHD wave in the corona (coronal wave hypothesis), which is reflected back into the chromosphere with different parts of the three-dimensional wave front successively intersecting the surface. The mathematical formulation of Uchida starts from the linearized set of MHD equations and derives an analog of the eikonal equation of geometrical optics with the hydromagnetic disturbance propagation problem. This derivation is set in a nonuniform anisotropic medium in the Wentzel-Kramers-Brillouin (WKB) approximation. Geometrical optics apply when the wavelength of the propagating disturbance is much smaller than the scale length of the inhomogeneities in the medium. Thus, a parallelism holds for the propagation of MHD waves in the WKB approximation.

In the WKB approximation, the motion of the wave packet is described as a "quasi-particle" and in the short-wavelength limit a hydromagnetic wave may be regarded as being propagated along rays that are refracted by the nonuniform medium. Since the density scale height in the corona $\left(5 \times 10^{4} \mathrm{~km}\right)$ is the shortest scale length in the equations of Uchida (1968), Uchida concludes that this approximation is valid in the corona for typical fast-mode waves.

For the simulation of the propagating fast-mode wave front we use the Eqs. (2a)-(2f) of Wang (2000) (Eqs. (1)-(6) below), where this latter author sets the fast-mode wave speed $u_{f}$ equal to the magnetosonic speed $\left(u_{A}^{2}+c_{s}^{2}\right)^{1 / 2}$, and not only the Alfvén speed as in Uchida et al. (1973), with $u_{A}=B /(4 \pi \rho)^{1 / 2}$ and $c_{s}=\left(\gamma K T / \mu m_{p}\right)^{1 / 2}$. Wang denotes $r$ as the heliocentric distance, $\theta$ the heliographic colatitude, $\phi$ the Carrington longitude, and $t$ the time. Additionally, if we neglect the effect of the orientation of the magnetic field with respect to the wave vectors and dissipation effects as well, the position and direction of propagation of a given point comoving with the fast-mode wave front can be determined by:

$r^{\prime}(t)=\kappa_{r} u_{f}$ 
$\theta^{\prime}(t)=\kappa_{\theta} u_{f} / r$

$\phi^{\prime}(t)=\kappa_{\phi} u_{f} /(r \sin \theta)$

$k_{r}^{\prime}(t) / k=-\partial u_{f} / \partial r+\left(\kappa_{\theta}^{2}+\kappa_{\phi}^{2}\right) u_{f} / r$

$k_{\theta}^{\prime}(t) / k=-(1 / r) \partial u_{f} / \partial \theta-\left(\kappa_{r} \kappa_{\theta}-\kappa_{\phi}^{2} \cot \theta\right) u_{f} / r$

$k_{\phi}^{\prime}(t) / k=-(1 / r \sin \theta) \partial u_{f} / \partial \phi-\left(\kappa_{r}+\kappa_{\theta} \cot \theta\right) u_{f} / r$,

where the prime denotes a time derivative and $\kappa_{r} \equiv k_{r} / k, \kappa_{\theta} \equiv$ $k_{\theta} / k$ and $\kappa_{\phi} \equiv k_{\phi} / k$.

Lastly, we evaluated our model on simple test cases (twolayer corona, stratified corona), performed resolution tests on the spatial step, and reproduced most of the ray-path plots in Wang (2000). These tests support the validity of the algorithm and we were able to move to the analysis of real data (for more details see Appendix C).

\subsection{Coronal background model}

To propagate the fast-mode MHD wave, it is necessary to compute the local magnetosonic speed $u_{f}$. This requires knowledge of the temperature, density, and Alfvén speed values of the coronal medium. For that, the output of a global 3D MHD coronal model is used.

We use 3D coronal models produced by the MHD Algorithm outside a Sphere (MAS) code ${ }^{1}$. The MAS code integrates the time-dependent resistive thermodynamic MHD equations in $3 \mathrm{D}$ spherical coordinates, solving them on a nonuniform logically rectangular staggered grid using finite differences ${ }^{2}$. The "thermodynamic" mode of MAS involves solving for additional terms that capture the thermodynamic energy balance in the low corona (coronal heating, radiative losses, electron heat conduction), while the nonuniformity of the grid enables MAS to efficiently resolve small-scale structures such as the transition region and active regions with a grid that spans the global solar corona from 1 to 20 or $30 R_{S}$. Each component of the field vectors is staggered, which, combined with the use of the vector potential as a primitive, guarantees that the magnetic field is divergence-free. To simulate observed conditions, photospheric magnetic field observations are used to specify the boundary condition on the radial magnetic field $\left(B_{r}\right)$. The code exhibits parallel strong scaling up to thousands of CPU cores on large HPC systems, and MAS is routinely used to model the structure of the solar corona and solar eruptions (e.g., Mikić et al. 1999, 2018; Lionello et al. 2009; Downs et al. 2013; Török et al. 2018).

In our case, the output from the MAS model is used to represent the undisturbed background state of the corona just before the eruptive event. Four different versions of the coronal model were examined in order to identify the one most suitable for this study and to evaluate the sensitivity of the ray tracing to the background conditions: hmi__cus-cor-poly-std $01^{3}$ is a simple polytropic MHD model that does not use the thermodynamic energy equation in the corona. Consequently, we used it only for some early tests and we do not retain it in our analysis. The other two models both use the same thermodynamic MHD code and boundary conditions (magnetic field maps from SDO/HMI), but specify the empirical coronal heating function differently. Heating Model 1 (hmi__med-cor-thermo1-std01) is the "com-

\footnotetext{
1 http://www . predsci. com/corona/model_desc.html

2 See Appendix A of Török et al. (2018) and (Caplan et al. 2017) for numerical details.

3 Results for the first three model types are publicly available: http: //www.predsci.com/mhdweb/data_access.php.
}

posite" model described in Lionello et al. (2009) and Heating Model 2 (hmi__med-cor-thermo2-std01) was developed by Zoran Mikić for the 2010 total solar eclipse prediction (Mikić et al. 2010).

The fourth and newest model is the wave-turbulence-driven (WTD) model $^{4}$, where coronal heating is achieved by the launch of low-frequency Alfvén waves in the chromosphere (Lionello et al. 2014; Downs et al. 2016; Mikić et al. 2018). In addition to its physical basis, a major advantage of the WTD approach is that few free parameters can be used to set the heating rate; instead the local heating rate adapts naturally to the strength and geometry of the 3D magnetic field (Downs et al. 2016). In practice, the range of temperature and density values produced by the WTD model lies roughly in-between those of the two previous models. The WTD model is also considered more advanced and able to represent with a higher accuracy the undisturbed coronal medium. Two WTD runs at low and high spatial resolution were used for each event under study. This model and specifically the high-resolution runs are the base of our analysis (for additional details about the different model comparison, see Appendix D). The input data for the WTD runs are based on SDO/HMI synoptic magnetograms. When the region of interest is close to the edge of the map then two merged HMI synoptic maps are used. The seam of the synoptic Carrington map is taken in such a way so that all data are within 2 weeks of the event of interest (this was the case for event 1 ; see Sect. 3.1). The merged map is then smoothed to the mesh resolution for the run. The maps of the high-resolution runs have more structure than those of the low-resolution runs, but both start from the exact same flux distribution. The low resolution runs have a $(219,98,203)$ mesh and the high-resolution runs a $(251,250,547)$ mesh, in the coordinates $(r, \theta, \phi)$. The radial dimension extends until 20 solar radii and has a logarithmic scale away from the transition region.

\subsection{Code - Visualization}

Our framework, which includes the numerical propagation of the fast-mode wave front, the initialization programs, the tests, and the visualization and analysis techniques, was developed in Python (Van Rossum 1995). The simulation of the propagating wave front takes as input the coronal data cubes from the MHD model, the three coordinates of the origin point of the EUV wave (constrained from observations and analysis, see Sect. 3), and a distribution of initial wave vectors that determines the initial directions of propagation. This distribution is taken as uniform over a sphere.

The output of this simulation is a collection of ray paths representing the successive spatial positions in time of the different wave vectors. For a given time, we can therefore derive a wave front. The wave front as well as the individual ray paths are visualized in 3D using Python libraries, specifically the Mayavi package (Ramachandran \& Varoquaux 2011).

To compare the simulated wave front with actual EUV observations, EUV spectroheliograms were mapped onto a 3D representation of the Sun bringing a high level of flexibility in the analysis process. As one of the two events was on the limb, the possibility to add planes representing the outer corona is included as well ("off-limb plane").

\footnotetext{
4 The WTD model is not yet run routinely, and so the data for generic cases are not publicly available.
} 


\subsection{Radio data}

Type II radio bursts are widely considered to be signatures of a shock in the solar corona (as discussed in Sect. 1) and since they are produced by plasma emission mechanisms, the frequency at any given time reflects the electron density where the driver is located. This is a very useful diagnostic tool that allows us, by tracking the frequency drift of the observed type II radio burst, to investigate the propagation of the source as it progressively moves to areas of lower density (typically higher in the corona). The plasma frequency is related to the electron density by

$f_{p}=C \sqrt{n_{e}}$,

where $C=8980 \mathrm{~Hz} \mathrm{~cm}^{3 / 2}$ is a constant.

Inversely, we can, from the simulation and the coronal model, deduce the electron density along each ray path and convert it into a temporal evolution of the frequency. It must be mentioned here that for this conversion from density to frequency we have made the calculation either for the harmonic frequency or the fundamental, simply based on the frequency at which the type II radio burst is most clearly observed.

To compare with the observations we implement the following approach: Investigation of which instantaneous position along each ray path could be responsible for the radio emission at the same moment. We identify the boundaries of the type II burst in the radio spectrum, fit third-degree polynomials to them, and then identify the ray-path points that are inside this frequency domain. With this method, instead of searching for a single ray path that can accurately reproduce the type II radio burst, we see which parts of the front could contribute to the type II radio burst emission. The ray-path points that encounter density values corresponding to frequencies within the type II radio burst delimited domain are displayed in red in our visualizations.

\subsection{Instruments}

During this study, data from many different instruments were used. For examination of the EUV wave kinematics and its comparison with the simulated fast-mode wave, we used images in EUV from SDO/AIA (193 $\AA$ ) and from PROBA2/SWAP (174 ̊).

The Atmospheric Imaging Assembly (AIA; Lemen et al. 2012) provides multiple simultaneous high-resolution full-disk images of the entire corona with 0.6 arcsec per pixel spatial resolution and $12 \mathrm{~s}$ cadence. The field of view (FOV) covered by AIA is $41 \operatorname{arcmin}\left(1.27 \mathrm{R}_{\odot}\right)$. The $193 \AA$ channel emission mainly comes from Fe XII and Fe XXIV lines, which have coronal origin and correspond to temperatures around 1.5 million degrees Kelvin (and up to 20 million for flares). The SWAP telescope (Sun Watcher using Active Pixel System detector and Image Processing; Seaton et al. 2013; Halain et al. 2013) provides images of the solar corona at about $174 \AA$, a bandpass that corresponds to a temperature of roughly 1 million degrees Kelvin, with a cadence of 1 image per 1-2 min, and a FOV of 54 arcmin.

For the analysis of the radio bursts during the two events, we used data from three different origins: the Humain station in Belgium, the Culgoora station in Australia, and the Nançay station in France. Data from Belgium come from two spectrographs, HSRS and ARCAS, covering in total a band from 45 to $\sim 1500 \mathrm{MHz}$. ARCAS (45-400 MHz) and HSRS (275$1495 \mathrm{MHz}$ ) produce dynamic spectra with time resolutions of $\sim 85$ and $\sim 250 \mathrm{~ms}$, respectively. The Culgoora station operates a solar radiospectrograph which sweeps through a frequency range of $18-1800 \mathrm{MHz}$ every three seconds. The Nançay Decameter Array (NDA; Lecacheux 2000) is a phased array spectrograph observing in the decametre range $(10-80 \mathrm{MHz})$ dedicated to the observation of the Sun and Jupiter, and routinely provides highly dynamic spectra with different receivers. For the current study, we used the one with a time resolution of $1 \mathrm{~s}$.

In order to constrain the coordinates of the EUV wave origin point, we analyzed data from the Reuven Ramaty High Energy Solar Spectroscopic Imager (RHESSI; Lin et al. 2002). RHESSI is designed to image solar flares in energetic photons from soft $\mathrm{X}$ rays $(\sim 3 \mathrm{keV})$ to gamma rays (up to $\sim 20 \mathrm{MeV}$ ) and to provide high-resolution spectroscopy up to gamma-ray energies. We were limited in the use of only the low-energy channel 6-12 keV, with a spatial resolution of 2 arcsec.

Additionally, X-ray flux data were used from GOES (Geostationary Operational Environmental Satellite). The GOES system, developed by NASA (National Aeronautics and Space Administration) and NOAA (National Oceanic and Atmospheric Administration), has a long history of operation, with many installments (newest GOES-17). Although its main use is for weather monitoring and forecasting, it is also equipped with a suite of instruments dedicated to space-weather operations. From GOES we used the X-ray sensor (XRS) in EXIS (Extreme Ultraviolet and X-ray Sensors), which provides data in the 1-8 and $0.5-4.0 \AA$ bands, with a cadence of $1 \mathrm{~s}$.

\section{Analysis and results}

The two events that we analyzed present two different geometry configurations, one edge-on (SOL2017-04-03T14:20:00) and one face-on (SOL2017-09-12T07:25:00). Our main analysis methods are the same for both events, and so we only detail the procedure for the first event and summarize the results for the second one.

\subsection{Event 1}

The first event that we studied happened on 3 April 2017. It took place in NOAA AR 12644 and was associated with an M5.6 flare and a CME. The flare started at 14:22 UT and was followed by an EUV wave around 14:24 UT that remained detectable for about 20 minutes. It was more visible on running difference images of the PROBA2/SWAP $174 \AA$ channel, which is one of the reasons why the data from this instrument were used for the analysis and as texture in the visualization scene. During that time, PROBA2 was off-pointed as part of an international campaign for coordinated observations (HOP 334$)^{5}$. As a result, the SWAP FOV covered an extended area where the wave propagated, and provided an opportunity to follow the erupting structures above the limb at higher altitudes than usual. During the event, a type II radio burst was observed from the Humain station in Belgium (Fig. 1). The type II radio burst started around 14:25 UT and drifted from $\sim 300$ to $45 \mathrm{MHz}$. Data from the NDA show it drifting even further down to $\sim 25 \mathrm{MHz}$.

In order to identify the coordinates of the point from which the EUV wave originated we combined the SWAP and RHESSI observations. In doing so, we make the assumption that the wave is triggered by a blast originating from the flare. From the reconstructed RHESSI image in the 6.0-12.0 keV range we have an estimation of the location and the spatial extent of the region from which the EUV wave could have originated. This specific

\footnotetext{
5 http://www.isas.jaxa.jp/home/solar/hinode_op/hop. php?hop=0334.
} 


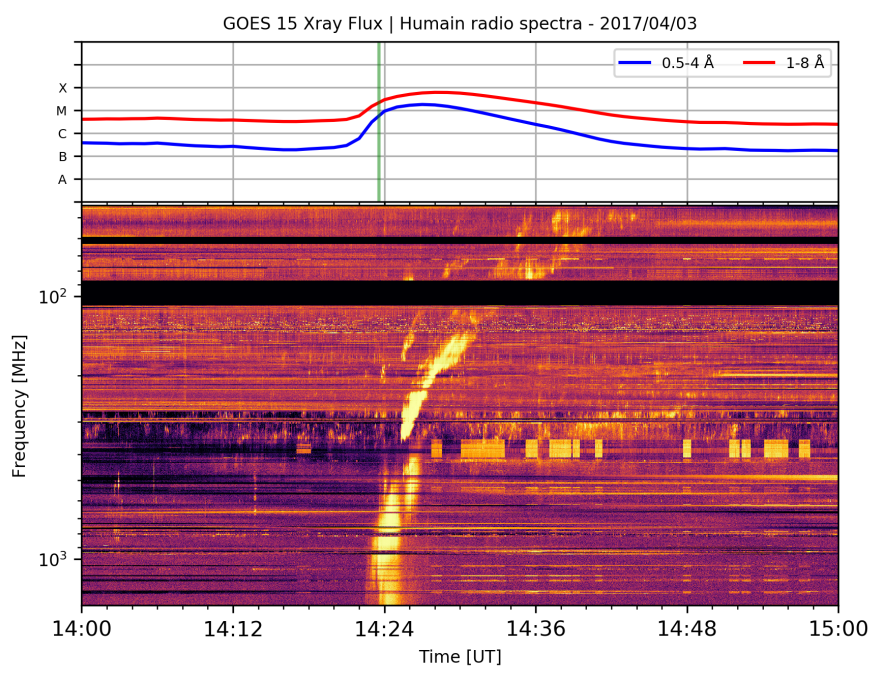

Fig. 1. Top: GOES $15 \mathrm{X}$-ray flux as measured during the M5.6 flare on 3 April 2017 (Event 1). The blue curve indicates the 0.5-4 A passband and the red curve the 1-8 A passband. The vertical green line marks the time of the RHESSI image in Fig. 2. Bottom: dynamic radio spectrum from the Humain station, showing the signature of the type II radio burst. The black horizontal stripes are bands filtered out or not sampled by the receivers.

energy range was selected because reconstructed images in other (higher) ranges were not available or were of poor quality. Additionally, because the X-ray emission is seen in projection over the solar disk, the exact height of the source is not known with certainty. Therefore, the RHESSI peak coordinates were deprojected, as if the source was located at different heights. This way a sample of "possible" origin points, derived from observations, was created. Figure 2 shows the origin points that were tested, on top of the local phase speed $\left(u_{f}\right)$ from the model, including the original RHESSI peak coordinates. Although this plot represents the local phase speed at a particular height in the solar corona, multiple heights were tested for almost every couple of longitude-latitude coordinates.

After comparing the positions of the possible origin points with the initial signature of the wave in EUV images and performing a phase speed analysis in the area of interest, in order to identify the points that allowed EUV wave propagation, the coordinates of these origin points were entered as input into the simulation and the computed fronts were examined. Since the propagation of the EUV wave depends on the gradient of the local magnetosonic speed (Wang 2000), $-\nabla u_{f}$, that it encounters, the propagation will be hindered or even stopped in areas with strong $u_{f}$ gradients. The phase speed analysis was used to find these areas and estimate the lowest possible height for the origin points (longitude-latitude pairs). This is seen in Fig. 2 (bottom), where two vertical lines indicate the lower limit for the propagation of an EUV wave (black dashed line) and the lower height of the possible origin points that were tested (red dashed line).

The selection of the de-projected RHESSI sources as the origin point did not provide the best qualitative match with the EUV observations. Additionally, a single best origin point could not be identified; a lot of origin points led to similar morphology of the propagation front and a number of different origin points were able to better reproduce different parts of the observed EUV signature. The AR 12644 showed significant evolution (flux emergence) near the limb a few days before the event (around April
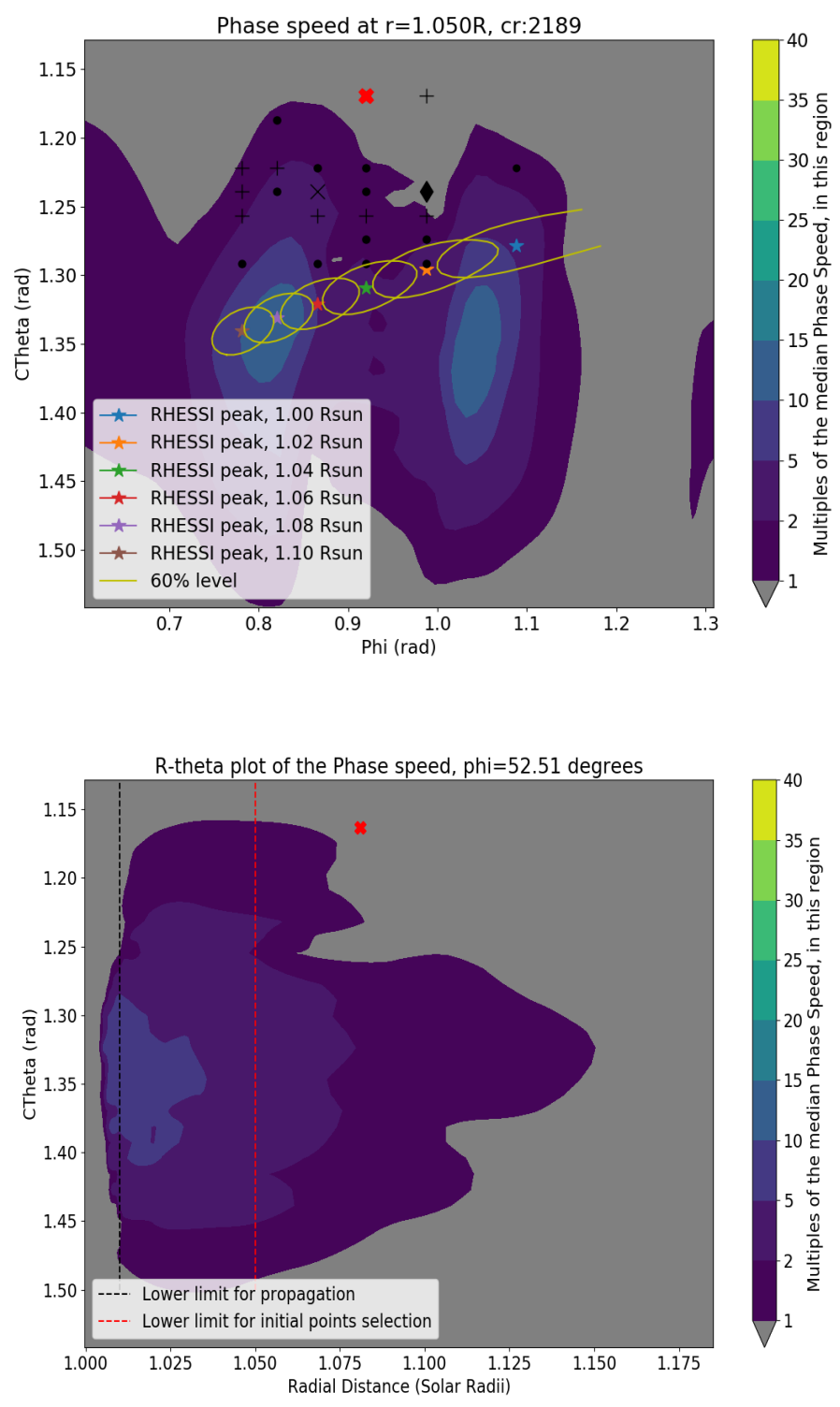

Fig. 2. Top: EUV wave origin points that were tested, on top of the local phase speed data, for Event 1 . The deprojected RHESSI peak coordinates (stars) together with the $60 \%$ contours (yellow) are also plotted, taking into account different source heights. The black marks of the points indicate the number of different heights that were tested (circle: 1, cross: 2, ex: 3, diamond: 4) and the point that was selected for the continuation of the analysis is indicated in red. Bottom: R-Theta plot of the local phase speed, with two vertical dashed lines indicating (black) the lower limit above which the wave can propagate and (red) the lower limit for most of the origin points that were tested. The red X symbol indicates the selected origin point.

1, 12:00 UT), and so when it erupted its topology was slightly different from that seen in the synoptic map, which is used as boundary conditions for the MHD model. This evolution a few days prior to the event could be responsible for the discrepancy induced by the two sets of origin point coordinates (those from the RHESSI de-projected sources and those producing a better match with the EUV wave signatures). Since in this work we try to reproduce the EUV wave with only one origin point, we selected one of the origin points, for which the observations matched the simulations to a significant degree, to continue our analysis. For more details on the motivation and caveats of our origin point selection, see Sect. 4 . 

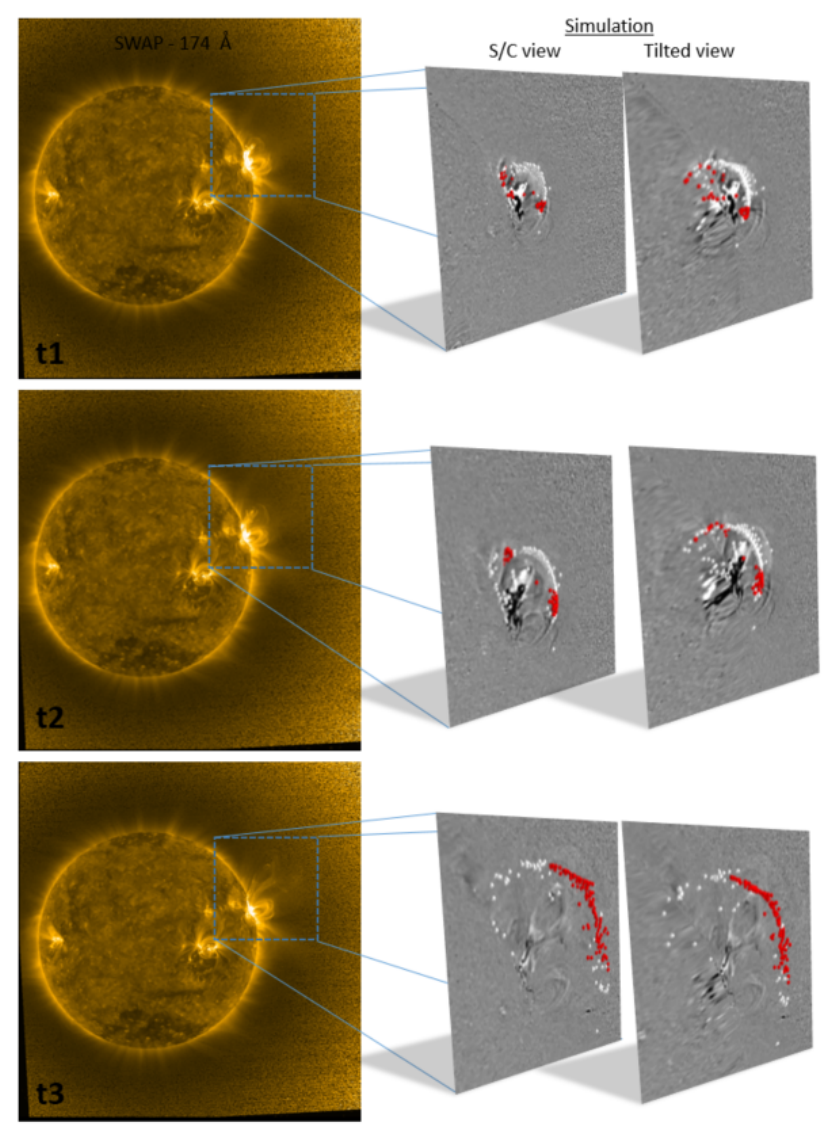

Fig. 3. Left: images of PROBA2/SWAP $174 \AA$ for three different times during Event 1 (from top to bottom: 14:26:10 UT, 14:28:00 UT, 14:37:10 UT). Right insets: zoom into the region of interest. The simulated wave front is displayed with white and red points on top of a background of PROBA2/SWAP running-difference images. Two different viewing angles for the same moment are shown, one that represents the PROBA2 viewing position and one tilted viewing position $\left(19^{\circ}\right.$ in azimuth) that was selected to optimize the visibility of the simulated wave front. The red points are identified candidates for the type II radio burst emission (see Sect. 2.4).

Figure 3 presents still frames from the visualization created for this event. The visualization frames in the right column of Fig. 3 are presented with some projection and shadow effects only for the visual "zooming" effect. The full movie is available online (Event 1 movie 1).

Figure 4 (left) shows the radio spectrum for this event, combining data from Humain and Nançay, including the selected boundaries for the type II radio burst and an indication of the three different times that correspond to the still frames of Fig. 3 (14:26:10 UT, 14:28:00 UT and 14:37:10 UT). The manually selected boundary points are displayed as blue crosses and the fitted curves as dashed black lines; for this, a dedicated program was developed, in which we can interactively select the boundary points and the fitting curve and immediately inspect the results on the displayed radio spectrum. Additionally, both upper and lower frequency boundaries can be extracted, so that they can be used from another program, which identifies the type II radio burst "candidate points" (red points in the figures), based on the conversion of the local density (MHD model) to frequency. As seen in Fig. 4, for this event the first harmonic lane of the type II radio burst signature was used.

Additionally, the angle between the wave vector $(\boldsymbol{K})$ and the magnetic field $(\boldsymbol{B})$ for every ray-path point was calculated from the dot product of the two vectors:

$\theta=\arccos \frac{\boldsymbol{B} \cdot \boldsymbol{K}}{|B||K|}$.

The wave vectors at each ray-path point are calculated from the numerical propagation of the EUV wave. But since in our numerical scheme the temporal step for every ray-path point depends on the local conditions it encounters, the time stamp (and consecutively that of the corresponding wave vector) is not the same for all the points. For that reason, all the ray-path points and their wave vectors are interpolated in time so that the position of all the ray-path points (wave vectors) can be retrieved at any given moment. The magnetic field components are derived from the MHD model and then interpolated on a regular grid. This way, the angle between the magnetic field and the wave vectors can be calculated for every spatial and temporal point inside the domain of our data set. The results (right plot in Fig. 4) show that for almost all the ray-path points whose density matches that of the source of the radio burst (in red), the corresponding wave vectors are quasi-perpendicular to the magnetic field.

Lastly, in order to more closely examine the localization of the radio source on the propagating wave front with respect to the dynamic radio spectrum, another visualization scene was created. Figure 5 presents a still frame of the corresponding movie, with the full movie available online (Event 1 movie 2).

The left and the right panels display two different views of the propagating wave front (point representation) at 14:39:54 UT. The points that are candidates for the type II radio burst emission are indicated in red. The middle panel displays the radio spectrum with the radio burst, the selected boundaries and a vertical black line which represents the position in time. This simultaneous view of the radio burst and the calculated source of the emission allows us to investigate the behavior of the type II radio burst source during the evolution of the EUV wave. The results show that different parts of the wave front could be responsible for the radio emission at different times. Initially, the source is located at the flanks (until 14:29 UT) but progressively moves to the nose of the front.

\subsection{Event 2}

The second event we investigated occurred on 12 September 2017. It took place in NOAA AR 12680 following a C3 class flare around 07:24 UT. An EUV wave is seen propagating to the south at approximately 07:25 UT for about $25 \mathrm{~min}$. During the event there was a type II radio burst that was best observed from the Culgoora (CSRS) station in Australia (Fig. 6). The type II radio burst started around 07:32 UT around $100 \mathrm{MHz}$ and lasted until 07:42 UT having drifted down to $20 \mathrm{MHz}$. Small eruptions were observed prior to the event around 06:15 UT and around 06:54 UT, but with no associated EUV waves.

In order to constrain the initial conditions of the simulation from observations, the same methodology as in Event 1 was followed. The reconstructed RHESSI image in the $6.0-12.0 \mathrm{keV}$ range (again, the only available) was analyzed together with the GOES flux curves of the flare and the EUV images (SDO/AIA$193 \AA$ ). It is noted here that the reconstructed RHESSI image (07:32-07:35 UT) is slightly after the flare peak (07:29 UT).

Figure 7 presents still frames from the visualization in the same layout as for Event 1. In the left column, SDO/AIA $193 \AA$ running difference images are now displayed. In the right column, two plots for different viewpoints combine observations and simulations for comparison. In this figure the "off-limb 

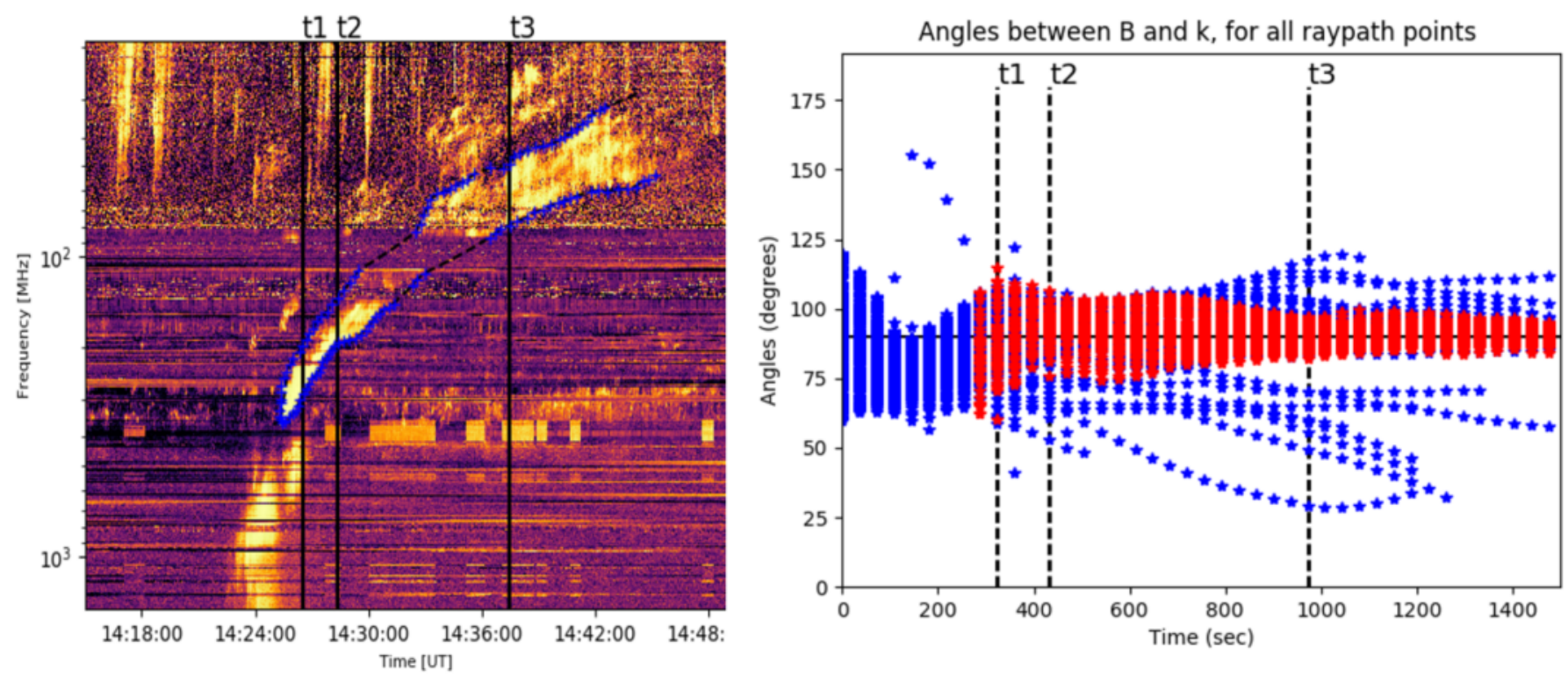

Fig. 4. Left: dynamic radio spectrum from the Humain station, showing the signature of the type II radio burst during Event 1 . The three vertical lines correspond to the time of the snapshots in Fig. 3. Blue crosses represent manually selected points and the dashed black lines the fitting curves. Right: angles between the magnetic field and the wave vector for every ray-path point. Red indicates the ray-path points that are identified as the potential source of the type II radio burst emission based on the local electron density matching the plasma frequency.

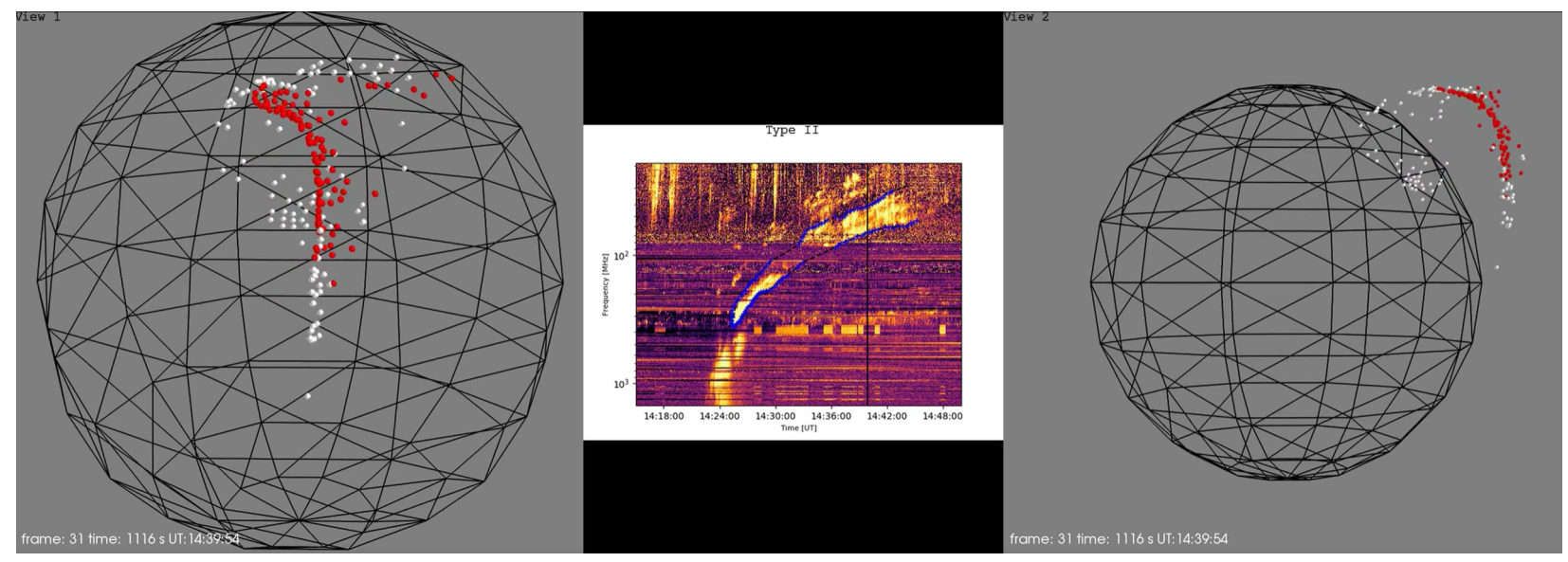

Fig. 5. Left and right: simulated wave front with white and red points on top of a spherical mesh representing the solar surface, in two viewing positions for Event 1 . The red points are identified as the potential source of the type II radio burst emission. Both panels represent the same moment in the simulation, which is shown in white in the bottom-left corner together with the UT time and the frame number of the movie (Event 1 movie 2). Middle: dynamic radio spectrum from the Humain and Nançay stations showing the type II radio burst signature and the selected boundaries. The vertical line represents the position in time in all the panels (14:39:54 UT).

plane" that was used in Event 1 is not included because the EUV wave propagates essentially towards the central part of the disk and there is no significant component visible in the offlimb EUV data. The points (white and red) represent the ray-path positions at different times with the red ones being the candidates for the type II radio burst emission (as described for Event 1 and in Sect. 2.4). The times of the still frames $(t 1, t 2, t 3)$ are respectively, 07:30:16 UT, 07:32:40 UT, and 07:34:30 UT. The complete movie can be found online (Event 2 movie 1). The results show a significant match between the simulated wave front and the observational EUV front, especially during the early stages of the propagation, for the RHESSI de-projected peak as origin point. In particular, despite the uniform distribution of the initial wave vector directions, very few ray paths propagate towards the east of the solar disk, which is consistent with the observed EUV wave.
Figure 8 presents the radio spectrum during the event, together with the type II radio burst boundaries and the indication of the three different time instances shown in Fig. 7. We note that for this event the selected boundaries belong to the fundamental frequency lane, since it is the one that is more clearly defined. As seen in the right plot of Fig. 8 and similarly to the first event, for all the ray-path points whose density matches that of the source position of the type II radio burst (red points), the wave vectors are quasi-perpendicular to the magnetic field.

Still frames from the simultaneous view of the radio spectrum and the simulated wave front are presented in Fig. 9, similar to the first event. The full movie is available online (Event 2 movie 2). Although the radio burst signature is shorter in duration than that of the previous event, the results show again that different parts of the wave front could be responsible for the radio emission at different times. Initially, the source is 


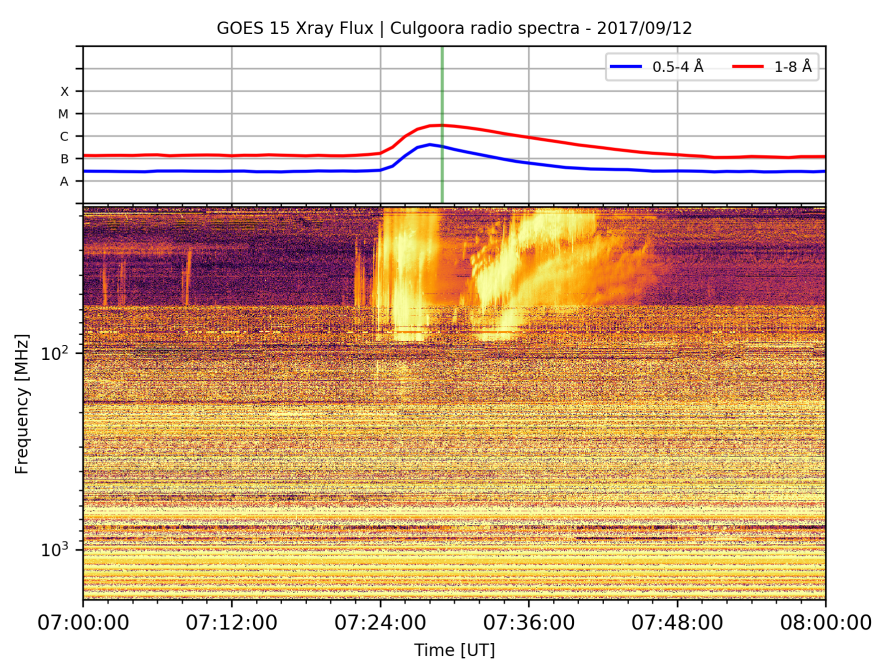

Fig. 6. Same as Fig. 1 for Event 2, except that the radio data are from the Culgoora station.
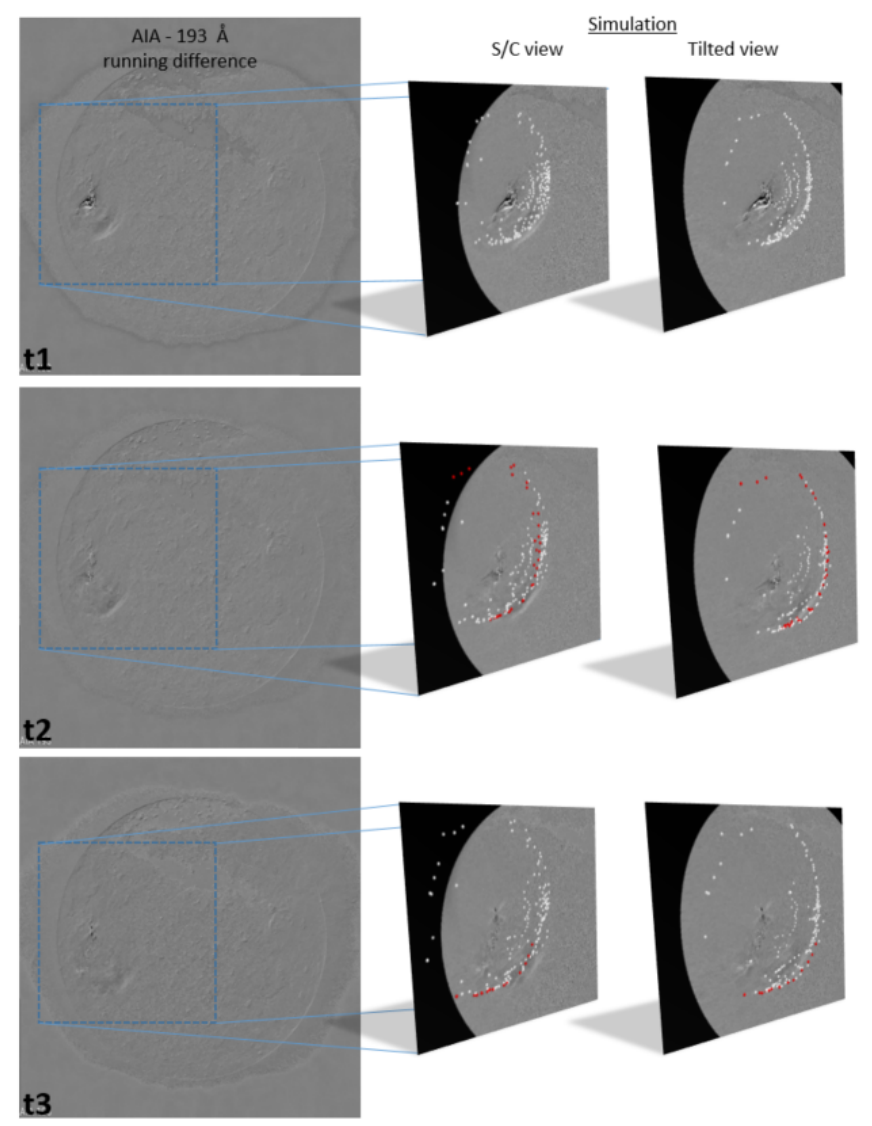

Fig. 7. Same as Fig. 3 for Event 2. However, the images are from the SDO/AIA-193A channel and in the visualization the EUV data are used only as texture for the solar surface and not for the off-limb region.

located in a narrow lane which encompasses almost the whole wave front but progressively moves to the southern part of the front.

\section{Discussion}

This work presents material supporting the idea that large-scale coronal waves signatures (enhanced EUV front and type II radio bursts) can be reproduced with the propagation of fast-mode waves. But the limitations of our framework must also be considered and discussed when interpreting these results.

First, the coronal conditions are simulated from an MHD model. Although this simulation is considered state-of-the-art, it still represents an approximation of the real conditions and especially of the undisturbed coronal medium. This approximation is more valid for the second event but not as accurate for the first event because of the dependence of the simulated coronal conditions on the photospheric magnetic field that is given as input. Particularly for the first event, we observe evolution inside and nearby the AR from the moment that the magnetic field is measured to the moment of the event and its position as it goes from on-disk (well observed) to off-limb (poorly observed; measurement depends on the line-of-sight component). This can heavily influence the ray-tracing results for a limb event. However, we expect this evolution to have an impact mostly on the magnetic and plasma conditions near the active region. The largescale behavior of the simulated wave front will remain mostly the same because it is based on the coronal conditions of a much larger domain. Nevertheless, this could explain why the best match between the simulation and the observations is obtained with an origin point situated away from the RHESSI source and the core of the active region: as the core was modified to a greater extent by the evolution, an origin point there is probably associated with more uncertainty. Starting with a point farther away avoids the complex magnetic structure on which a small modification can lead to very different wave propagation. Furthermore, provided that the real wave front eventually passes through the less disturbed "origin" point that we selected, a similar wave front can be formed later (Huygens-Fresnel principle).

Furthermore, we consider the soft X-ray (the only available energy range) signature in the reconstructed RHESSI images as the source location of the EUV wave. This signature includes thermal emission that spans a large portion of the loop and is not restricted to the loop top. One would expect the loop top to be a more suitable site for the origin of the EUV wave because it is closer to the reconnection site (Klein et al. 1999). This implies that the RHESSI peak coordinates provide only a rough estimate of the origin point and should not be considered as the absolute source location of the EUV wave.

Additionally, the fact that different origin points match different parts of the wave front to a greater or lesser extent could be an indication that in reality the observed EUV wave originates not from a single point but from the sum of multiple points. This would be the case for a piston-driven wave generated by the rapid transverse expansion of the erupting flux system. In this scenario, the EUV wave becomes freely propagating relatively early on as super-radial expansion slows (e.g., Patsourakos \& Vourlidas 2009; Downs et al. 2012; Vršnak et al. 2016, and review by Long et al. 2017). This would imply an effective surface of origin points near to the eruption site at different times. However, because the rate of expansion is still influenced by the local phase speed distribution, this effect would be difficult to distinguish from a few origin sites localized near the initiation site (i.e., the flare).

On the other hand, in the blast wave scenario, a physical interpretation of multiple origins could be that, amidst the complex magnetic topology of the active region core, the initial wave found different "exit" points from the active region. In light of these physical complexities and the systematic challenges described above, it is not surprising that no perfect single origin point can be found with the simulation. In a sense, this justifies our flexible, data-constrained approach to the problem. 

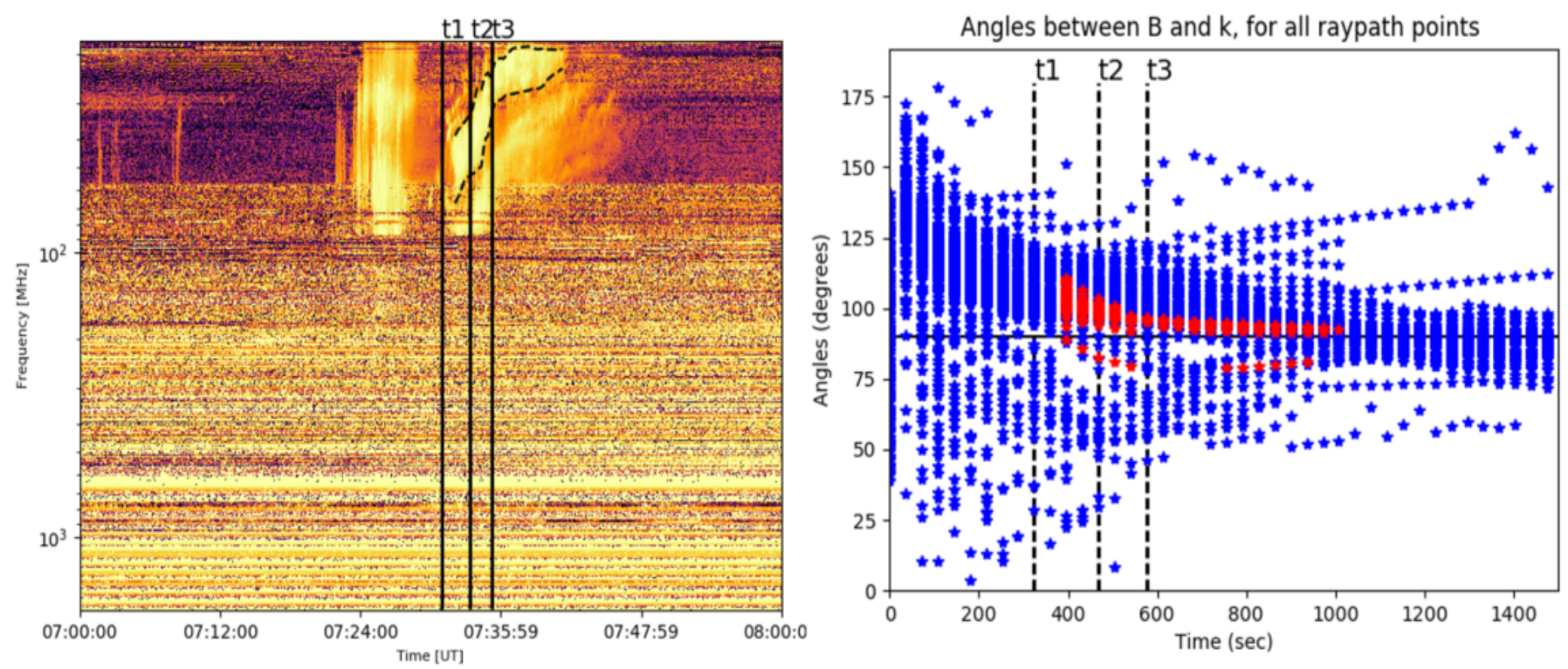

Fig. 8. Same as Fig. 4 for Event 2, except that the dynamic radio spectrum is from the Culgoora station. The times of the vertical lines (07:30:16 UT, 07:32:40 UT, 07:34:30 UT) match the time of the snapshots in Fig. 7.
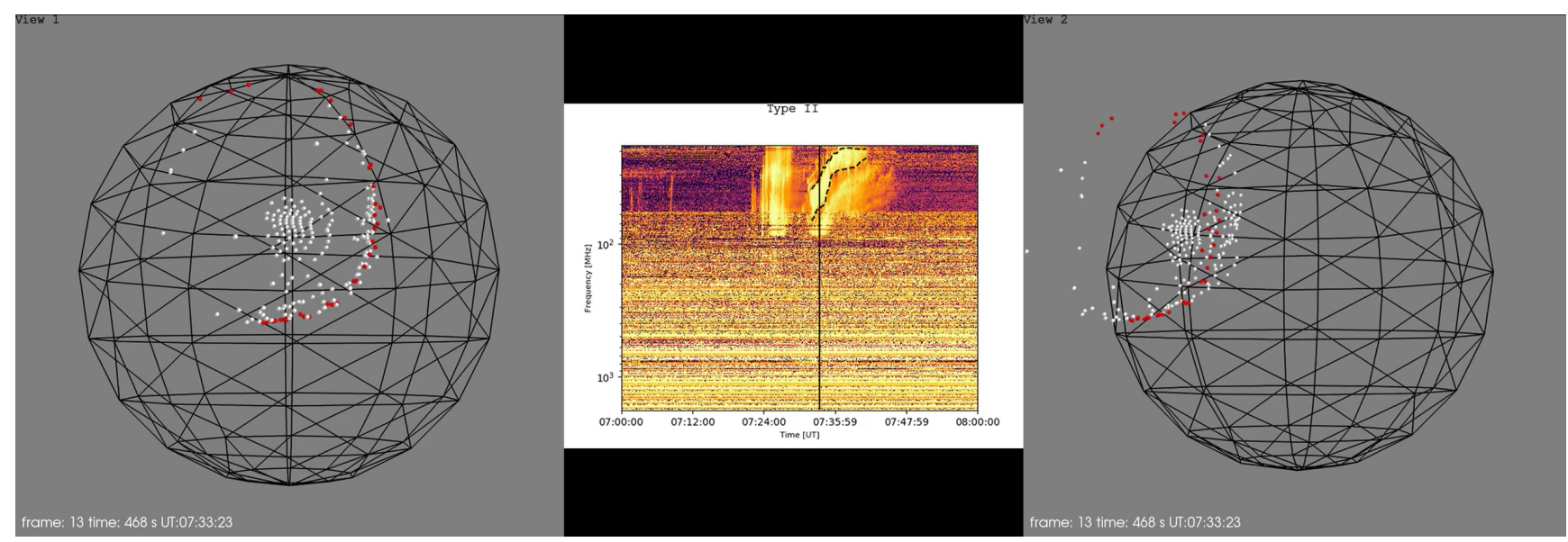

Fig. 9. Left and right: same as Fig. 5 for Event 2. Middle: dynamic radio spectrum from the Culgoora station showing the type II radio burst signature and the selected boundaries. The vertical line represents the position in time in all the panels (07:33:23 UT).

Nonlinear effects are not included in the numerical propagation of the fast-mode wave. Specifically, we use the formalism of Wang (2000), where neither dissipation nor the effect of the orientation of the magnetic field with respect to the wave vector are taken into consideration. However, these simplifications, as Wang (2000) pointed out, will not have a significant impact on the general behavior of the fast mode, because the magnetosonic speed $u_{f}$ only weakly depends on them (at most a factor $2^{1 / 2}$ in the case of the orientation of the magnetic field).

Additionally, we propagate a fast-mode wave and do not model a real shock, which would be necessary for the production of the observed type II radio bursts. The reason why this simplification may still be valid is that the coronal shocks correlated with EUV-waves and type II radio bursts are considered weak shocks (Warmuth et al. 2004b). In fact, by assuming a simple fast mode wave and not a shock, we are not describing any energy dissipation, nor do we treat the amplitude of the wave, but we focus mainly on the overall kinematic behavior.

Despite the aforementioned constraints, this framework shows that even a quite simple ray-path fast-mode model is able to reproduce the EUV wave observations to a significant degree. More importantly, we show that it is possible to localize the source of the type II radio burst on the propagating wave front. This is of great importance, because the position of the type II radio burst source is debated (Cliver et al. 1999; Nitta et al. 2014; Zucca et al. 2014). Furthermore, we show that the possible type II radio burst source position on the wave front is not constant in time but changes throughout the propagation, and that different parts of the front can be responsible for the emission at different times, especially during the early stages. In other words, as the wave front propagates and expands, it will encounter diverse local conditions and these local conditions are the ones that will dictate whether or not the subsequent electron acceleration leads to type II radio emission. By tracking the local conditions of the wave front in regions where the encountered density matches the density derived from the radio observations, our analysis shows that the angle between the wave vector and the local magnetic field can be considered as one of the necessary conditions. Although for both events the overlying magnetic topology confirms this dependency, this is 
particularly apparent for event 1 , because the overlying geometry of the field is that of a streamer. At the beginning of the radio event, the quasi-perpendicular configuration (discussed in more detail below) is more likely to be seen on the flank, and later on as the wave propagates outward it will start to see the overlaying arcades of the base of the streamer and the emission is more likely to be seen at the nose of the wave front. However, spectral radio imaging would be needed to accurately follow the evolution of the type II radio burst source position, if any. It remains unclear whether or not the source motion would be detectable at all given the rather typical low spatial resolution of instruments able to do this (e.g., LOFAR, MWA); although a recent case study observed with the Nançay Radioheliograph (Mancuso et al. 2019) suggests they would. This analysis is out of the scope of the current work but is considered as a necessary step in the future.

Lastly, our framework shows that almost all the ray-path points that are considered as the potential source of the type II radio burst emission (red points) display a quasi-perpendicular configuration between the wave vectors and the magnetic field. Although theoretical works (Mann et al. 1995) show that type II radio bursts can form either in quasi-perpendicular or quasiparallel configurations under different conditions, there is a lot of supporting evidence that the quasi-perpendicular configuration is favored and shocks accelerate particles efficiently in these topologies (Carley et al. 2013). This is due to the fact that the efficiency of the main mechanism for particle acceleration in coronal shocks, the shock drift acceleration (SDA), favors quasi-perpendicularity (Holman \& Pesses 1983). A more detailed investigation on the mechanism that creates the type II radio burst emission is out of the scope of this paper, but our results support the quasi-perpendicular configuration for type II radio bursts.

\section{Conclusions}

Here, we present the analysis of two EUV (EIT) wave events on 3 April 2017 and 12 September 2017. This analysis is based on a qualitative match between the EUV observations and a numerically propagated linear fast-mode MHD wave using the ray-tracing method. The plasma and magnetic field parameters of the coronal medium are derived from a global MHD simulation. Additionally, the type II radio bursts occurring during these events were investigated in order to identify which ray-path points are associated with densities that match the frequency of the type II radio burst signature. Our conclusions can be summarized as follows.

- Even a fairly simple ray-path model of fast-mode MHD waves displays a good qualitative match with the observations.

- The location of the type II radio burst emission on the propagating wave front can be derived for the duration of the event.

- The position of the type II radio burst source on the wave front is not stationary but evolves with time, meaning that different areas of the wave front can be responsible for different parts of the radio burst signature.

- The wave vectors of the ray-path points that are identified as the potential source of the radio type II burst emission are quasi-perpendicular to the magnetic lines.

This study serves as an initial examination of a framework for the analysis of the association between EUV (EIT) waves and type II radio bursts, and is planned to be extended in the future, specifically: by refining the fast-mode model (with nonlinearities such as dissipation and dampening) and by the comparison of the localized type II radio burst source with radio images (radioheliographs Nancay, LOFAR), which will be the main validation test for the aforementioned results.

Acknowledgements. This work was supported by the Research Foundation Flanders (FWO), PhD-aspirant grant $(1180919 \mathrm{~N})$ and partially by the PROBA2 Guest Investigator Program in the Royal Observatory of Belgium. We also gratefully acknowledge a 1-month visitor's grant of the not-for-profit (ASBL-VZW) association "Dynamics of the Solar System" for Alexandros Koukras. C. M. wishes to thank Y.-M. Wang for early discussions about EUV waves and fast mode MHD waves and for sharing the code from his seminal paper on that topic The authors acknowledge the Nançay Radio Observatory / Unite Scientifique de Nançay of the Observatoire de Paris (USR 704-CNRS, supported by Université d'Orléans, OSUC, and Région Centre in France) for providing access to NDA observations accessible online at http://www.obs-nancay.fr We are grateful to the Australian Bureau of Meteorology, Space Weather Services for the provision of the Culgoora spectrograph data. The solar instruments at the Humain radio astronomy station are supported by the Solar Terrestrial Center of Excellence.

\section{References}

Afanasyev, A. N., \& Uralov, A. M. 2011, Sol. Phys., 273, 479

Athay, R. G., \& Moreton, G. E. 1961, ApJ, 133, 935

Attrill, G. D. R., Harra, L. K., van Driel-Gesztelyi, L., \& Démoulin, P. 2007, ApJ, 656, L101

Biesecker, D. A., Myers, D. C., Thompson, B. J., Hammer, D. M., \& Vourlidas, A. 2002, ApJ, 569, 1009

Caplan, R. M., Mikić, Z., Linker, J. A., \& Lionello, R. 2017, J. Phys. Conf. Ser., 837, 012016

Carley, E. P., Long, D. M., Byrne, J. P., et al. 2013, Nat. Phys., 9, 811

Chen, P. F., \& Fang, C. 2011, ArXiv e-prints [arXiv:1108. 5274]

Chen, P. F., Wu, S. T., Shibata, K., \& Fang, C. 2002, ApJ, 572, L99

Chen, P. F., Fang, C., \& Shibata, K. 2005, ApJ, 622, 1202

Cliver, E. W., Webb, D. F., \& Howard, R. A. 1999, Sol. Phys., 187, 89

Delaboudinière, J. P., Artzner, G. E., Brunaud, J., et al. 1995, Sol. Phys., 162, 291

Delannée, C., \& Aulanier, G. 1999, Sol. Phys., 190, 107

Delannée, C., Török, T., Aulanier, G., \& Hochedez, J. F. 2008, Sol. Phys., 247, 123

Dere, K. P., Brueckner, G. E., Howard, R. A., et al. 1997, Sol. Phys., 175, 601

Downs, C., Roussev, I. I., \& Van Der Holst, B. 2011, ApJ, 728, 2

Downs, C., Roussev, I. I., van der Holst, B., Lugaz, N., \& Sokolov, I. V. 2012, ApJ, 750, 134

Downs, C., Linker, J. A., Mikić, Z., et al. 2013, Science, 340, 1196

Downs, C., Lionello, R., Mikić, Z., Linker, J. A., \& Velli, M. 2016, ApJ, 832, 180

Eto, S., Isobe, H., Narukage, N., et al. 2002, PASJ, 54, 481

Gallagher, P. T., \& Long, D. M. 2011, Space Sci. Rev., 158, 365

Halain, J. P., Berghmans, D., Seaton, D. B., et al. 2013, Sol. Phys., 286, 67

Holman, G. D., \& Pesses, M. E. 1983, ApJ, 267, 837

Klassen, A., Aurass, H., Mann, G., \& Thompson, B. J. 2000, A\&AS, 141, 357

Klein, K. L., Khan, J. I., Vilmer, N., Delouis, J. M., \& Aurass, H. 1999, A\&A, 346, L53

Krupar, V., Magdalenić, J., Eastwood, J. P., et al. 2019, ApJ, 882, 92

Kumar, P., Cho, K. S., Chen, P. F., Bong, S. C., \& Park, S. H. 2013, Sol. Phys., 282, 523

Lecacheux, A. 2000, Geophys. Monograph Ser., 119, 321

Lemen, J. R., Title, A. M., Akin, D. J., et al. 2012, Sol. Phys., 275, 17

Lin, R. P., Dennis, B. R., Hurford, G. J., et al. 2002, Sol. Phys., 210, 3

Lionello, R., Linker, J. A., \& Mikić, Z. 2009, ApJ, 690, 902

Lionello, R., Velli, M., Downs, C., et al. 2014, ApJ, 784, 1

Liu, W., \& Ofman, L. 2014, Sol. Phys., 289, 3233

Long, D. M., Bloomfield, D. S., Chen, P. F., et al. 2017, Sol. Phys., 292, 7

Magdalenić, J., Marqué, C., Krupar, V., et al. 2014, ApJ, 791, 115

Mancuso, S., Frassati, F., Bemporad, A., \& Barghini, D. 2019, A\&A, 624, L2

Mann, G., Classen, T., \& Aurass, H. 1995, A\&A, 295, 775

McKerns, M., \& Aivazis, M. 2010, Pathos: A Framework for Heterogeneous Computing

McKerns, M. M., Strand, L., Sullivan, T., Fang, A., \& Aivazis, M. A. G. 2012, ArXiv e-prints [arXiv:1202.1056]

Mikić, Z., Linker, J. A., Schnack, D. D., Lionello, R., \& Tarditi, A. 1999, Phys. Plasmas, 6, 2217

Mikić, Z., Linker, J. A., Lionello, R., Riley, P., \& Titov, V. S. 2010, AGU Fall Meeting Abstracts

Mikić, Z., Downs, C., Linker, J. A., et al. 2018, Nat. Astron., 2, 913 


\section{A. Koukras et al.: EUV wave connection with type II burst}

Moreton, G. E., \& Ramsey, H. E. 1960, PASP, 72, 357

Moses, D., Clette, F., Delaboudinière, J. P., et al. 1997, Sol. Phys., 175, 571

Müller, D., Nicula, B., Felix, S., et al. 2017, A\&A, 606, A10

Nelson, G. J., \& Melrose, D. B. 1985, in Solar Radiophysics: Studies of Emission

From the Sun at Metre Wavelengths, eds. D. J. McLean, \& N. R. Labrum, 333

Newkirk, G., Jr 1961, ApJ, 133, 983

Nindos, A., Aurass, H., Klein, K. L., \& Trottet, G. 2008, Sol. Phys., 253, 3

Nitta, N. V., Liu, W., Gopalswamy, N., \& Yashiro, S. 2014, Sol. Phys., 289, 4589

Ofman, L., \& Thompson, B. J. 2002, ApJ, 574, 440

Patsourakos, S., \& Vourlidas, A. 2009, ApJ, 700, L182

Patsourakos, S., \& Vourlidas, A. 2012, Sol. Phys., 281, 187

Ramachandran, P., \& Varoquaux, G. 2011, Comput. Sci. Eng., 13, 40

Saito, K., Poland, A. I., \& Munro, R. H. 1977, Sol. Phys., 55, 121

Seaton, D. B., Berghmans, D., Nicula, B., et al. 2013, Sol. Phys., 286, 43

Shen, Y., \& Liu, Y. 2012, ApJ, 752

Shen, Y., Liu, Y., Su, J., et al. 2013, ApJ, 773, 2

Shen, Y., Chen, P. F., Liu, Y. D., et al. 2019, ApJ, 873, 22

Thompson, B. J., Gurman, J. B., Neupert, W. M., et al. 1999, ApJ, 517, L151

Thompson, B. J., Plunkett, S. P., Gurman, J. B., et al. 1998, Geophys. Res. Lett., 25,2465

Török, T., Downs, C., Linker, J. A., et al. 2018, ApJ, 856, 75

Uchida, Y. 1968, Sol. Phys., 4, 30
Uchida, Y. 1970, PASJ, 22, 341

Uchida, Y., Altschuler, M. D., \& Newkirk, G. J. 1973, Sol. Phys., 28, 495 Van Rossum, G. 1995, CWI Report, CS-R9526, 1

Veronig, A. M., Temmer, M., \& Vršnak, B. 2008, ApJ, 681, L113

Virtanen, P., Gommers, R., Oliphant, T. E., et al. 2020, Nat. Methods, 17, 261

Vršnak, B., Žic, T., Lulić, S., Temmer, M., \& Veronig, A. M. 2016, Sol. Phys., 291, 89

Wang, Y.-M. 2000, ApJ, 543, L89

Warmuth, A., \& Mann, G. 2011, A\&A, 532, A151

Warmuth, A., Vršnak, B., Aurass, H., \& Hanslmeier, A. 2001, ApJ, 560, L105

Warmuth, A., Vršnak, B., Magdalenić, J., Hanslmeier, A., \& Otruba, W. 2004a, A\&A, 418, 1101

Warmuth, A., Vršnak, B., Magdalenić, J., Hanslmeier, A., \& Otruba, W. 2004b, A\&A, 418, 1117

West, M. J., Zhukov, A. N., Dolla, L., \& Rodriguez, L. 2011, ApJ, 730, 122

Wild, J. P., \& McCready, L. L. 1950, Aust. J. Sci. Res. A Phys. Sci., 3, 387

Wu, S. T., Zheng, H., Wang, S., et al. 2001, J. Geophys. Res. Space Phys., 106, 25089

Zhukov, A. N. 2011, J. Atmos. Sol.-Terr. Phys., 73, 1096

Zhukov, A. N., \& Auchère, F. 2004, A\&A, 427, 705

Zucca, P., Carley, E. P., Shaun Bloomfield, D., \& Gallagher, P. T. 2014, A\&A, 564, A 47 


\section{Appendix A: Visualization}

We describe here how the visualization of the computed EUV wave was achieved. The purpose of the visualization is to examine the kinematics of a simulated EUV wave and compare them with observations in EUV. It was therefore necessary to know its position with respect to the surface of the Sun. To this aim, a 3D representation of the surface of the Sun was implemented in a 3D scene and EUV images were added to it as a texture. The 3D scene was created using the Mayavi and tvkt libraries from Python and the EUV images (SDO/AIA, PROBA2/SWAP) were retrieved from JHelioviewer (Müller et al. 2017). This provided the ability to reload the EUV image that was the texture of the Sun's surface through a decorator function, and to combine the observations with the numerically propagated wave front in real time. Because one of the events that we selected for further analysis was positioned on the West Limb (Event 1) and propagated outwards, there was a need to compare with the EUV observations above the limb. For that, an "off-limb plane" was added in the 3D scene: a plane that cuts the sphere representing the Sun and on which we projected only the off-limb part of the EUV images. Initially, the representation of the wave front was achieved with a mesh (made using 3D triangulation) but the complex topology made the interpretation difficult (for the reader). For that reason, we decided to use the "points" representation of the wave front, where we display only the ray-path points at every moment and not connections between them.

\section{Appendix B: Numerical scheme}

In order to numerically propagate the fast-mode, we integrate Eqs. (1)-(6) to obtain the wave-front position as a function of time. For that, the Python package SciPy (Virtanen et al. 2020) is used. In particular, the scipy.integrate.odeint routine, which solves the initial value problem for systems of first-order ordinary differential equations using lsoda from the FORTRAN library odepack.

The initial conditions that must be specified are the coordinates of the origin point (radial distance, latitude, longitude), the three components of its wave vector $\left(k_{0_{r}}, k_{0_{p h i}}, k_{0_{\text {theta }}}\right)$, and the number of points in time for which the integration will be calculated. The coordinates of the origin point are derived from observations and the components of the wave vector from a dedicated program that creates a uniform distribution of wave vectors in a sphere. This way the EUV wave is initialized without a preferential direction of propagation but with a uniform distribution of wave vectors over all directions, as would be expected from a blast origin mechanism.

Lastly, the evaluation of the code for the numerical propagation was made parallel using the Python package Pathos (McKerns \& Aivazis 2010; McKerns et al. 2012) in order to be able to handle a large number of wave vectors. This provided one order of magnitude increase in the computation speed.

\section{Appendix C: Tests and validation}

Before applying the wave-front propagation model to the analysis of real events, we validated it on simple test cases. One of these was to create simple configurations of the coronal atmosphere and identify if the ray-path propagation happens according to theoretical expectations. Specifically, we created a two-layered corona with two distinct regions of different Alfvén speed values and compared the refraction index derived from the ray-path angles with the one expected from the Snell-Descartes

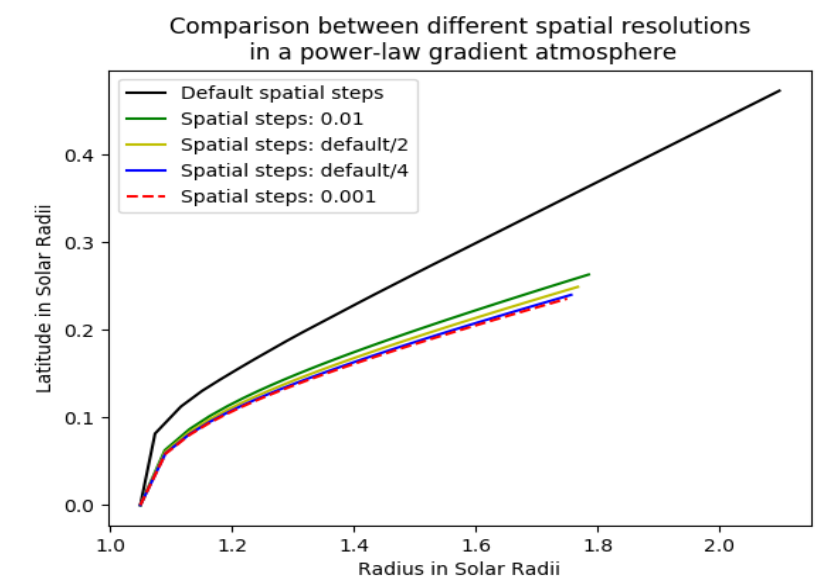

Fig. C.1. Effect of spatial resolution on a ray-path trajectory starting with the same origin point, with differently colored lines indicating the use of different spatial steps. The ray-path propagation was made in a stratified atmosphere.

law. The other simple case was a stratified corona (both with a selected gradient and a gradient defined from real data). For both cases, the relative error between our model and the theoretically expected value of the refracting angles was below $0.8 \%$ and as low as $0.1 \%$.

Consecutively, we also performed spatial resolution tests in order to investigate the dependence of the ray-path trajectories with the spatial step in our computation. In the reduction of the spatial step, we made a compromise between the significant trajectory improvement and the substantial increase of the computation time. This can be seen in Fig. C.1, where a ray path is drawn using different spatial steps. The continuous decrease of the spatial step does not affect the ray-path trajectory after some point, but this is not the case for the computational cost, which keeps increasing. The final spatial step that was selected is shown with the red curve.

Additionally, different methods were examined for the interpolation of the MHD data: nearest neighbor and linear. The linear method showed better results and was selected for the rest of the analysis.

Lastly, we tried to replicate the results of Wang (2000) for the events on the 7 of April and 12 of May 1997 (both Earth-directed halo CMEs). We were able to accurately reproduce his results with the only exception a particular ray path on 12 of May (figure 5 in Wang (2000)). Even using the same magnetic field data as input (from Wilcox Solar Observatory) the difference persisted. We interpreted this discrepancy as resulting from the different spatial resolutions of the coronal data models and possibly the details of the calculation of the wave path itself. All the above tests supported the validity of our algorithm.

\section{Appendix D: MHD model comparison}

During this study different versions of the coronal MHD model were tested. This includes versions that vary in sophistication and also in resolution (see Sect. 2.2). Below we present the results of some of these tests, using the high-resolution version of the WTD model as a reference point.

One of the first comparisons that we implemented was between the $\mathrm{hmi}$ _-med-cor-thermo2-std 01 model, hereafter referred to as Model 3, and the WTD model in the highresolution version. As metrics for the similarity of the two models, we calculated the relative difference of the norm of the 


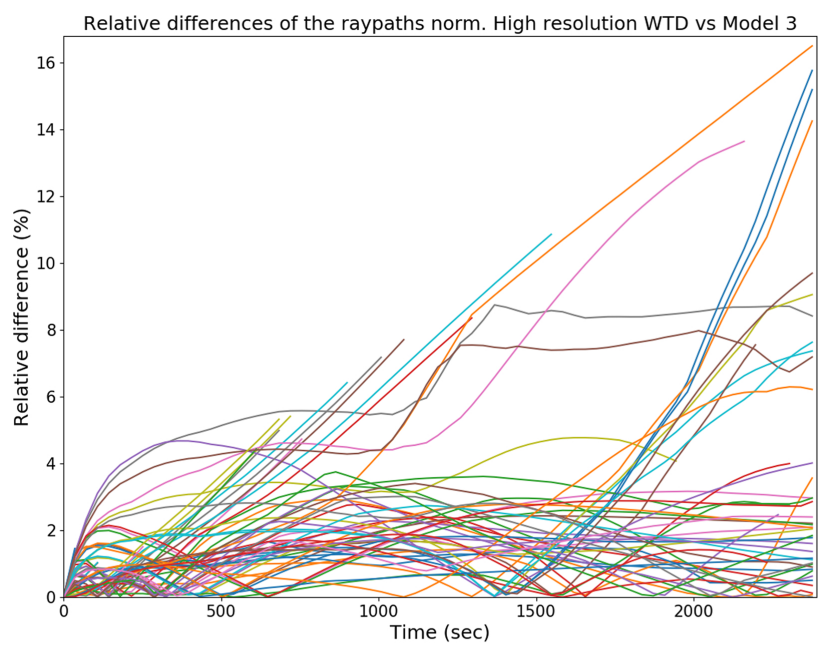

Mean relative difference of the raypaths at everytime step. High resolution WTD vs Model 3

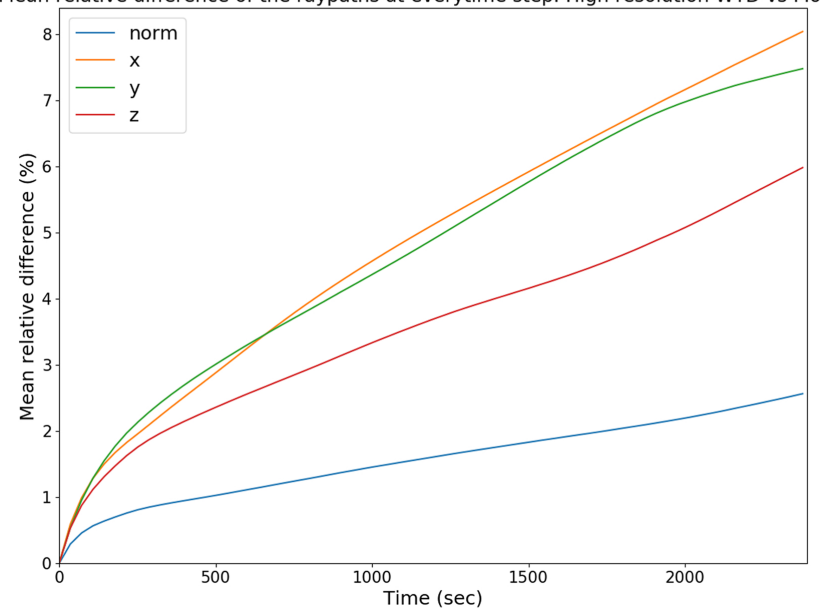

Fig. D.1. Top: percentage of the relative difference between the norm of the position for every ray path. The different colors represent different ray paths. The comparison is made between the high-resolution WTD model and Model 3. Bottom: percentage of the mean relative difference amongst the ray paths of the high-resolution WTD model and Model 3. This metric is shown for the three spatial components and the norm, with the colors given in the legend.

instant position for every ray path, along with the mean relative difference of the norm and of the three spatial components $(\mathrm{x}, \mathrm{y}, \mathrm{z})$ (inside the visualization scene the transformation from Spherical to Cartesian coordinates is applied), as show in Fig. D.1. The behavior that is observed is that only a small number of ray paths differ significantly $(>10 \%)$, with the majority of them being below $4 \%$. The mean relative difference increases with time, which is expected because small differences in the underlying coronal conditions will lead to bigger deviations as the wave evolves. This discrepancy between the two models is more prominent for $\mathrm{x}, \mathrm{y}$, and $\mathrm{z}$ directions than for the norm and it reaches around $8 \%$ at the end of the simulation. Although, these relative differences present a quantitative measure for the comparison, they cannot display the general behavior of the two waves. For that reason, the creation of a movie displaying the propagation of both waves simultaneously is used. A still frame of that movie is presented in Fig. D.2. The full movie is available online (High res WTD - Model 3). Examining the movie, it is clear that the two waves show significant differences in their propagation, which is not surprising given the differences in heating models leading to different 3D density distributions and

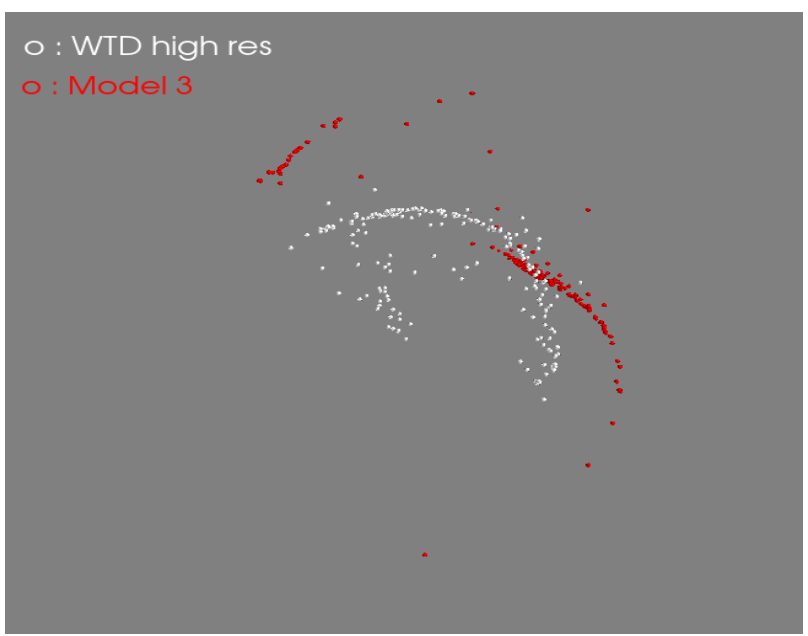

Fig. D.2. Still frame of a movie showing the propagation of two EUV waves in two different coronal models. The positions of the ray paths that compose the wave front are represented as points. White shows the high-resolution WTD model and red shows Model 3. This frame is taken at 900 seconds of the simulation. The full movie is available online (High res WTD - Model 3).
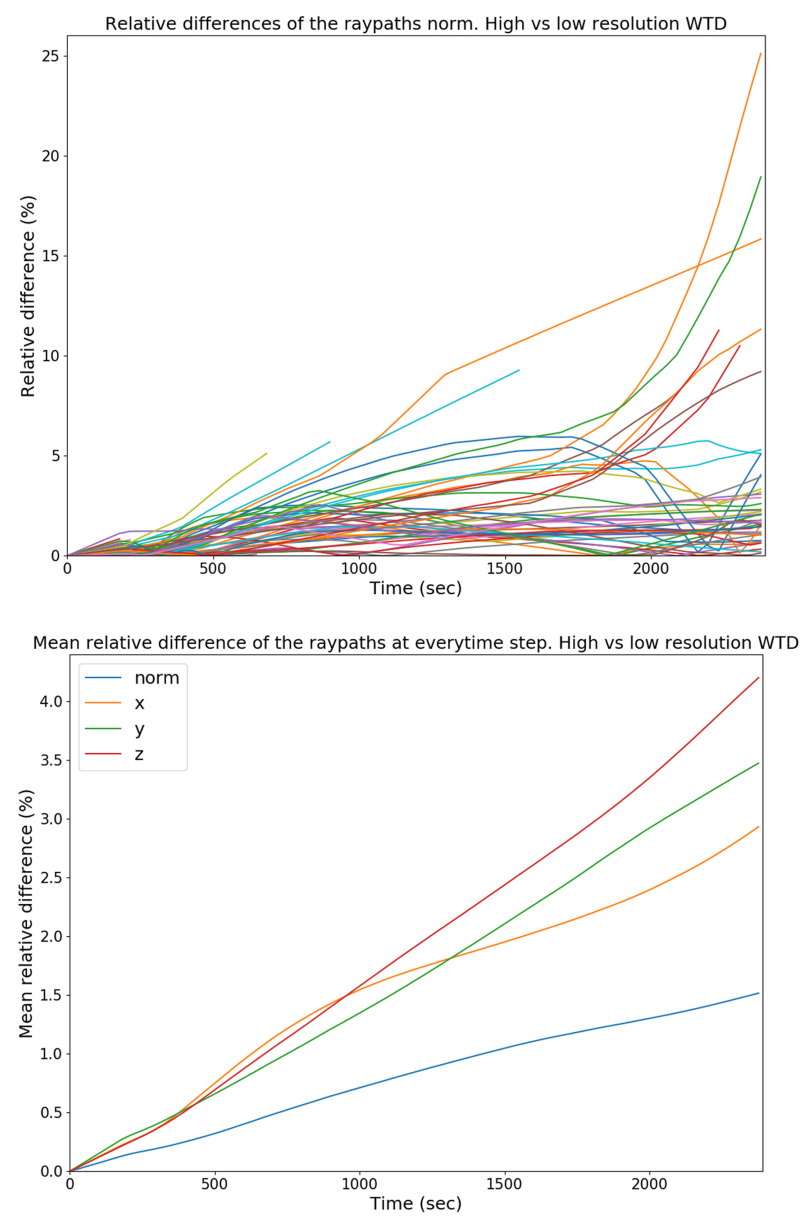

Fig. D.3. Same as Fig. D.1 but the comparison is between the high- and low-resolution WTD models.

thus local phase speeds. The wave in Model 3 presents a faster propagation during the early stages of the simulation and a separation in two distinct components in the later stages. Although there are some signatures of a northern component in the EUV 


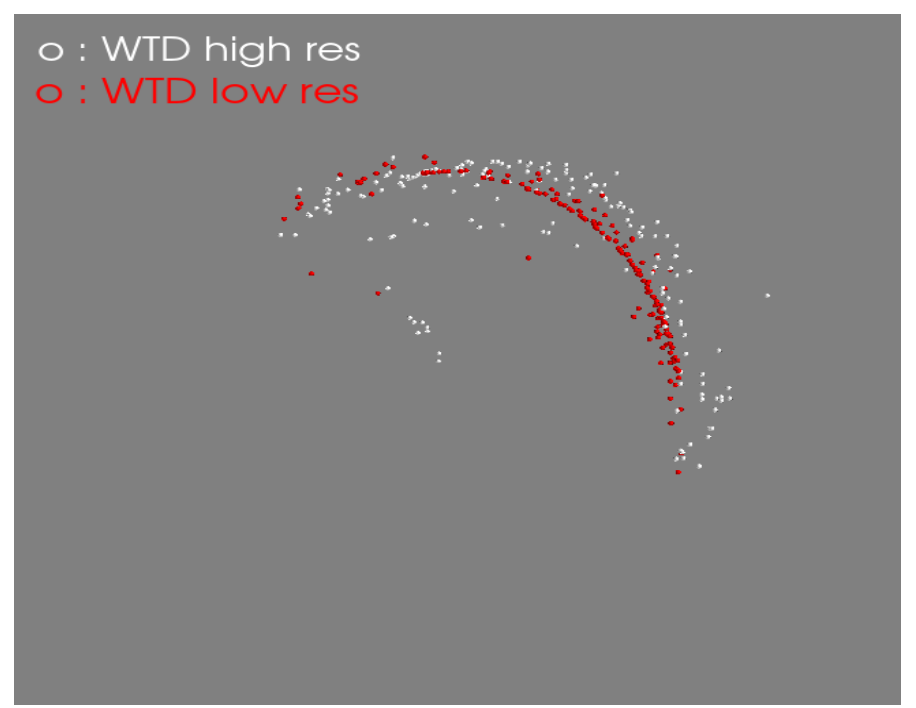

Fig. D.4. Same as Fig. D.2, but the red points now represent the lowresolution WTD model. This frame is taken at 1440 seconds of the simulation. The full movie is available online (High res WTD - Low res WTD).

observations, the overall behavior correlates to a lower degree with the observational data compared with the wave simulated in the high-resolution WTD model.

The next model comparison that was implemented is between the high- and low-resolution WTD models in order to additionally investigate the effect of resolution on the propagation of the wave. The same tests as in the previous case were used. The relative difference between the ray paths and the mean relative difference are shown in Fig. D.3. It is clear that the majority of the ray-path relative differences display lower values than the previous comparison and only two ray paths show very large discrepancies. Additionally, the mean relative difference is a factor two lower for all components, in comparison with the previous case. These results prove that the two waves share a greater similarity compared to the waves in Model 3 and in the high-resolution WTD. This is expected because the coronal models in question are only different in terms of resolution. A movie was create to take a closer look at the general behavior of the waves, which includes the simultaneous propagation of both, as before. A still frame of that movie is displayed in Fig. D.4 and the complete movie is available online (High res WTD - Low res WTD). A closer examination of the movie shows that the two waves present similar propagation and shape. The only minor differences are that the wave in the high-resolution WTD model appears to be slightly faster, and at later stages of the evolution its wave front is more diffuse than the one in the low-resolution WTD model, which displays a more compact wave front.

These tests clearly indicate that the WTD model provides coronal medium conditions that better match the observations. Additionally, the difference in resolution has a small effect on the general propagation and shape of the wave front, but a higher resolution model will give rise to more realistic wave front behavior. Based on the above, we select the high-resolution WTD model for our simulations and analysis. 